\section{Risco de suicídio e comportamentos de risco à saúde em jovens de 18 a 24 anos: um estudo descritivo}

\author{
Suicide risk and health risk behavior among youth \\ between the ages of 18 and 24 years: \\ a descriptive study
}

\author{
Liliane da Costa Ores 1 \\ Luciana de Avila Quevedo ${ }^{1}$ \\ Karen Jansen 1 \\ Adriana Bezerra de Carvalho 1 \\ Taiane Azevedo Cardoso 1 \\ Luciano Dias de Mattos Souza 1 \\ Ricardo Tavares Pinheiro 1 \\ Ricardo Azevedo da Silva 1
}

\section{Abstract}

1 Universidade Católica de Pelotas, Pelotas, Brasil.

Correspondência

R. A. Silva

Universidade Católica de Pelotas.

Rua Almirante Barroso 1202, Pelotas, RS 96010-280, Brasil. ricardo.as@uol.com.br
The aim of this study was to assess suicide risk and risk behavior in young people. A cross-sectional study in the urban area of Pelotas, Rio Grande do Sul State, Brazil was performed using systematic sampling with young people between the ages of 18 to 24 years. We measured the risk of suicide (MINI), behavior (YRBSS), abuse/substance dependence (ASSIST) and socioeconomic status (ABEP). Data was analyzed using SPSS software. The sample consisted of 1,560 young people and the prevalence of suicide risk was 8.6\%. Suicide risk was associated with: having suffered an accident that required a visit to the emergency room ( $p=0.011)$, fighting $(p=0.016)$, carrying a weapon $(p=0.001)$ and carrying $a$ firearm ( $p \leq 0.001)$, substance abuse/dependence ( $p \leq 0.001)$, not having used a condom during the last sexual relationship ( $p=0.025)$, not having $a$ steady partner $(p \leq 0.001)$ and having sex with five or more people ( $p=0.018)$. Young people that present risk behavior also represent a suicide risk.

Suicide; Risk Taking; Young Adult

\section{Introdução}

A juventude é um período em que estrutura-se a identidade, sendo assim, uma etapa do desenvolvimento que exige mudanças nos níveis sociais, familiares, físicos e afetivos. Essas mudanças, embora normais, levam o jovem a experimentar crescente ansiedade e angústia, aumentando o risco de problemas emocionais, entre os quais sintomas depressivos e risco de suicídio parecem estar entre os mais preocupantes 1,2,3,4.

A transformação da adolescência para a vida adulta também é uma etapa em que comportamentos de risco, como a participação em atividades que possam comprometer a saúde física e mental do jovem estão presentes 5 . Muitas dessas condutas podem iniciar apenas pelo caráter exploratório do jovem, assim como pela influência do meio; entretanto, caso não sejam precocemente identificadas, podem levar à consolidação destas atitudes com significativas consequências nos níveis individual, familiar e social ${ }^{4}$. A ocorrência do comportamento suicida entre jovens aparenta estar aumentando nas últimas décadas, e a adolescência destaca-se como o período mais relacionado à morte por causas externas, como o suicídio 6,7.

Comportamento suicida envolve, esporádica ou frequentemente, ideias, desejos e manifestações da intenção de querer morrer, planejamento de como, quando e onde fazer isso, além do 
pensamento de como o suicídio irá impactar os outros, muitas vezes, como solução para algo insuportável e insolúvel. Constitui, portanto, uma tendência autodestrutiva que se apresenta em um gradiente de gravidade que pode variar da ideação ao suicídio 1,2. O risco de suicídio em si abrange desde a ideação suicida até tentativas cometidas. A avaliação do risco de suicídio continua sendo um desafio aos profissionais da saúde e, geralmente mais importante do que buscar a causa do suicídio de imediato.

Um estudo realizado com jovens do Ensino Médio demonstrou que $12,1 \%$ pensaram em suicídio e 11,5\% haviam planejado o suicídio ${ }^{8}$. Estudo de base populacional com uma amostra de 1.560 jovens, de 18 a 24 anos da zona urbana de Pelotas, Rio Grande do Sul, mostrou que a prevalência de risco de suicídio entre jovens foi de $8 \%$. Essas taxas justificam a crescente atenção que vem sendo dada mundialmente ao tema e muitos países têm procurado desenvolver estratégias nacionais para a prevenção 10 .

O presente estudo tem como objetivo avaliar a relação entre comportamentos de risco à saúde e risco de suicídio em jovens de 18 a 24 anos, da zona urbana de Pelotas, tendo em vista a busca de estratégias de prevenção de mortes.

\section{Método}

Estudo transversal de base populacional com jovens de 18 a 24 anos de idade, residentes em Pelotas. A seleção amostral foi realizada por conglomerados, no período de agosto de 2007 a dezembro de 2008, considerando a população de 39.667 jovens e a divisão censitária atual de 408 setores na zona urbana da cidade de Pelotas (Instituto Brasileiro de Geografia e Estatística. Censo Demográfico 2000. http://www.ibge.gov.br). A fim de garantir a inclusão da amostra necessária, 89 setores censitários foram sorteados sistematicamente. A seleção dos domicílios nos setores sorteados foi realizada segundo uma amostragem sistemática, sendo o primeiro domicílio a residência da esquina pré-estabelecida pelo Instituto Brasileiro de Geografia e Estatística (IBGE) como início do setor, o intervalo de seleção foi determinado por um pulo de dois domicílios entre os sorteados. Quando identificados mais de um jovem elegível na residência, todos eram convidados a participar do estudo.

Os critérios de inclusão foram ter entre $18 \mathrm{e}$ 24 anos, ter capacidade cognitiva para entender e responder os instrumentos e aceitar a participação no estudo assinando um Termo de Consentimento Livre e Esclarecido. A coleta de dados foi realizada por meio de visita domiciliar, nesse momento foram coletados os dados sobre comportamentos de risco à saúde e risco de suicídio. Os comportamentos de risco entre os jovens foram avaliados com uso do Youth Risk Behavior Surveillance System (YRBSS) 11. O YRBSS monitora seis categorias de comportamentos de risco à saúde entre jovens e adultos: (1) comportamentos que contribuem para as lesões não intencionais e violência; (2) uso de tabaco; (3) uso de álcool e outras drogas; (4) comportamentos sexuais que contribuem para a gravidez indesejada e doenças sexualmente transmissíveis (DST), incluindo o vírus da imunodeficiência humana (HIV); (5) comportamentos alimentares insalubres e (6) inatividade física. As categorias utilizadas neste estudo foram comportamentos que contribuem para as lesões não intencionais de violência e comportamentos sexuais. Dentro da categoria violência, foi avaliado o comportamrento de risco no trânsito e envolvimento em brigas. Os comportamentos de risco relacionados à sexualidade foram avaliados por questões referentes ao uso de preservativo na última relação sexual, ingestão de bebida alcoólica antes da última relação, não ter parceiro fixo e o número de pessoas que teve relação sexual nos últimos 12 meses.

O abuso ou dependência de substâncias foram avaliados com utilização do Alcohol, Smoking and Substance Involvement Screening Test (ASSIST) 12, que é um instrumento que tem o objetivo de mensurar os hábitos relativos ao uso de drogas lícitas (álcool e tabaco) e ilícitas (maconha, solventes, cocaína, loló, cola, crack, inalantes, tranquilizantes, estimulantes, anfetamina, sedativos, alucinógenos e opiáceos). Adaptado e validado para o Brasil, e incluídas questões referentes à substância de consumo inicial e idade da primeira experimentação.

A avaliação do risco de suicídio, transtorno de ansiedade generalizada, transtorno bipolar e episódio depressivo maior foi realizada pela Mini Internacional Neuropsychiatric Interview (MINI). Esta entrevista estruturada e de curta duração é destinada à utilização na prática clínica e de pesquisa, e visa classificar os entrevistados de forma compatível com os critérios de eixo I do DSM-IV e da 10 a revisão da Classificação Internacional de Doenças (CID-10) 13. O módulo sobre suicídio questiona diversos componentes do risco de suicídio pelas seguintes questões: durante o último mês - (1) "Você já pensou que seria melhor estar morto ou desejou estar morto?" (escore: 1 ponto); (2) "Você já quis prejudicar a si mesmo?" (2 pontos); (3) "Você já pensou em cometer suicídio?” (6 pontos); (4) "Você já planejou como cometer suicídio?" (10 pontos); (5) "Você já tentou suicídio?" (10 pontos); e (6) "Alguma vez ao longo da vida você já tentou o suicídio?” (4 pontos). O risco de suicídio foi classificado em "baixo” (escore 1-5), 
"moderado" (escore 6-9) e "alto" (escore $\geq 10$ ). Para a análise, os escores foram dicotomizados em ausência de risco (baixo ou ausente) e presença de risco (risco moderado ou alto), como recomendado pelos autores da MINI, portanto, uma resposta de 6 pontos ou mais, foi considerada risco de suicídio 14 .

Foi utilizada a classificação da Associação Brasileira de Empresas de Pesquisa (ABEP) para avaliar o nível socioeconômico das famílias. Essa classificação é baseada no acúmulo de bens materiais e na escolaridade do chefe da família, classificando os sujeitos em cinco níveis (A, B, C, D e E) 15 .

Os instrumentos foram codificados e digitados com dupla entrada no programa Epi Info 6.04d (Centers for Disease Control and Prevention, Atlanta, Estados Unidos) e checagem automática no momento da digitação; além disso, foi testado no mesmo programa a consistências da digitação comparando as duas entradas de dados. A análise dos dados foi realizada no programa SPSS (SPSS Inc., Chicago, Estados Unidos). Foram calculadas as razões de prevalência com intervalos de $95 \%$ de confiança e procedeu-se às análises bivariadas mediante o teste do quiquadrado.

O projeto foi aprovado pelo Comitê de Ética em Pesquisa da Universidade Católica de Pelotas (UCPel), de acordo com o protocolo 2006/96, e os jovens que apresentaram risco de suicídio foram encaminhados ao Ambulatório de Psiquiatria do Campus Olivé Leite da UCPel.

\section{Resultados}

Foram identificados 1.762 jovens de 18 a 24 anos, mas $202(11,5 \%)$ jovens se recusaram a participar do estudo. Assim, a amostra final foi constituída de 1.560 jovens com idade média de 20,5 $( \pm 2,1)$ anos. Desses, $56,4 \%$ (880) do gênero feminino, $73,3 \%$ (1.144) de cor da pele branca e 48,1\% (751) pertenciam à classificação socioeconômica C segundo ABEP.

A prevalência de risco de suicídio entre os jovens avaliados foi de 8,6\%. Desses, 48 (31,6\%) tinham transtorno de ansiedade generalizada, 75 $(37,7 \%)$ transtorno bipolar e $72(36,7 \%)$ apresentavam episódio depressivo maior. A presença de qualquer um desses transtornos teve associação significativa com o risco de suicídio ( $\mathrm{p} \leq 0,001)$.

Entre os jovens que apresentaram risco de suicídio, 9,8\% não utilizaram o cinto de segurança, 8,9\% não utilizaram o capacete, $6,3 \%$ ultrapassaram o sinal vermelho, $6,9 \%$ dirigiram ou andaram na carona com motorista bêbado, 12,6\% sofreram acidente que os obrigou a ir ao pronto- socorro, 18,4\% ingeriram bebida alcoólica antes de se acidentar (Tabela 1).

Em relação a comportamentos violentos de risco à saúde, dos jovens que apresentaram risco de suicídio, 13,9\% entraram em briga com agressão física, 29,2\% carregaram arma branca nos últimos 30 dias, 55,6\% carregaram arma de fogo nos últimos 30 dias (Tabela 2).

Entre os jovens com risco de suicídio, 15,4\% eram dependentes de tabaco, 13,4\% faziam uso abusivo de álcool, $26 \%$ eram dependentes de maconha, 30,3\% dependentes de cocaína, 37,5\% dependentes de crack, $40 \%$ fizeram uso abusivo de anfetaminas e $34,4 \%$ usavam sedativos de forma abusiva (Tabela 3).

Quanto ao comportamento de risco à saúde relacionado à sexualidade, 10,5\% não usaram preservativo na última relação sexual, 10,3\% ingeriram bebida alcoólica antes da última relação sexual, 7,9\% não tinham parceiro fixo e 10\% tiveram relação sexual com cinco ou mais pessoas nos últimos 12 meses (Tabela 4).

Estiveram relacionadas com o risco de suicídio as seguintes variáveis: sofrer acidente que o tenha obrigado a ir ao pronto-socorro $(p=0,011)$, entrar em briga com a agressão física $(p=0,016)$, carregar arma branca nos últimos 30 dias $(\mathrm{p}=$ $0,001)$, carregar arma de fogo nos últimos 30 dias ( $\mathrm{p}<0,001)$, dependência de tabaco $(\mathrm{p}<0,001)$, uso abusivo de álcool ( $\mathrm{p}<0,001)$, dependência de maconha ( $\mathrm{p}<0,001)$, de cocaína $(\mathrm{p}<0,001)$, de $\operatorname{crack}(\mathrm{p}=0,001)$, dependência de anfetamina ( $p=0,001)$, e de sedativos ( $p<0,001)$. Quanto aos comportamentos sexuais, foram associados ao risco de suicídio não ter usado preservativo na última relação sexual ( $\mathrm{p}=0,025)$, não ter tido parceiro fixo $(\mathrm{p}<0,001)$ e ter tido relação sexual com cinco ou mais pessoas no último ano $(\mathrm{p}=0,018)$.

\section{Discussão}

Este estudo sugere a relação entre risco de suicídio e comportamentos de risco em jovens. Comportamentos de risco à saúde podem estar relacionados ao risco de suicídio como consequência de atos inconscientes. Na adolescência já é esperado um comportamento de risco maior, contudo, quando há risco de suicídio, os comportamentos de risco tornam-se mais intensos.

Este estudo verificou a associação entre acidentes de trânsito e risco de suicídio. Tal relação pode estar muitas vezes subestimada por conta de a intenção suicida estar disfarçada nessa situação. De acordo com os estudos sobre o tema, cerca de $1,5 \%$ a quase $15 \%$ de todas as fatalidades no trânsito são suicídios 16 . 
Comportamentos de risco à saúde no trânsito, nos últimos trinta dias. Pelotas, Rio Grande do Sul, Brasil.

\begin{tabular}{|c|c|c|c|c|c|}
\hline & \multirow[t]{2}{*}{$\mathrm{n}$} & \multirow[t]{2}{*}{$\%$} & \multicolumn{2}{|c|}{ Risco de suicídio } & \multirow[t]{2}{*}{ Valor de $\mathrm{p}$} \\
\hline & & & $\mathrm{n}$ & $\%$ & \\
\hline \multicolumn{6}{|c|}{ Uso do cinto de segurança } \\
\hline Não & 469 & 30,1 & 46 & 9,8 & 0,317 \\
\hline Sim & 1.087 & 69,9 & 88 & 8,1 & \\
\hline \multicolumn{6}{|c|}{ Uso do capacete } \\
\hline Não & 527 & 33,8 & 47 & 8,9 & 0,831 \\
\hline Sim & 1.030 & 66,2 & 87 & 8,5 & \\
\hline \multicolumn{6}{|c|}{ Ultrapassou sinal vermelho } \\
\hline Não & 774 & 79,1 & 60 & 7,8 & 0,59 \\
\hline Sim & 205 & 20,9 & 13 & 6,3 & \\
\hline \multicolumn{6}{|c|}{ Dirigiu ou andou na carona com motorista bêbado } \\
\hline Não & 1.278 & 82,2 & 115 & 9 & 0,3 \\
\hline Sim & 277 & 17,8 & 19 & 6,9 & \\
\hline \multicolumn{6}{|c|}{ Sofreu acidente que o obrigou a ir ao Pronto-Socorro } \\
\hline Não & 1.271 & 81,7 & 98 & 7,7 & 0,011 \\
\hline Sim & 285 & 18,3 & 36 & 12,6 & \\
\hline \multicolumn{6}{|c|}{ Ingeriu bebida alcoólica antes de se acidentar } \\
\hline Não & 246 & 86,6 & 29 & 11,8 & 0,378 \\
\hline Sim & 38 & 13,4 & 7 & 18,4 & \\
\hline Total & 1.560 & 100,0 & 134 & 8,6 & \\
\hline
\end{tabular}

Tabela 2

Comportamentos violentos de risco à saúde, nos últimos trinta dias. Pelotas, Rio Grande do Sul, Brasil.

\begin{tabular}{|c|c|c|c|c|c|}
\hline & \multirow[t]{2}{*}{$\mathrm{n}$} & \multirow[t]{2}{*}{$\%$} & \multicolumn{2}{|c|}{ Risco de suicídio } & \multirow[t]{2}{*}{ Valor de $p$} \\
\hline & & & n & $\%$ & \\
\hline \multicolumn{6}{|c|}{ Entrou em briga com agressão física } \\
\hline Não & 1.390 & 89,3 & 111 & 8 & 0,016 \\
\hline Sim & 166 & 10,7 & 23 & 13,9 & \\
\hline \multicolumn{6}{|c|}{ Carregou arma branca } \\
\hline Não & 1.500 & 98,4 & 124 & 8,3 & 0,001 \\
\hline Sim & 24 & 1,6 & 7 & 29,2 & \\
\hline \multicolumn{6}{|c|}{ Carregou arma de fogo } \\
\hline Não & 1.530 & 99,4 & 128 & 8,4 & $\leq 0,001$ \\
\hline Sim & 9 & 0,6 & 5 & 55,6 & \\
\hline Total & 1.560 & 100,0 & 134 & 8,6 & \\
\hline
\end{tabular}

Brigas com agressões físicas também foi um fator relacionado ao risco de suicídio neste estudo. Existe a evidência de que altos níveis de agressão durante a vida são associados com alto risco de suicídio ${ }^{17}$. A associação encontrada no presente estudo pode, talvez, ser justificada porque agressão física é uma conduta de risco violenta que pode expor o indivíduo a danos severos.

No Brasil, o método mais utilizado em suicídios consumados, em todas as idades, é o enforcamento $(55,7 \%)$ seguido por arma de fogo $(13,2 \%)$ e ingestão de pesticida $(5,5 \%) 18$. O presente estudo verificou alta prevalência de jovens 
Tabela 3

Uso de substâncias psicoativas, nos últimos três meses. Pelotas, Rio Grande do Sul, Brasil.

\begin{tabular}{|c|c|c|c|c|c|}
\hline & \multirow[t]{2}{*}{$\mathrm{n}$} & \multirow[t]{2}{*}{$\%$} & \multicolumn{2}{|c|}{ Risco de suicídio } & \multirow[t]{2}{*}{ Valor de $p$} \\
\hline & & & $\mathrm{n}$ & $\%$ & \\
\hline \multicolumn{6}{|l|}{ Tabaco } \\
\hline Sem uso/Ocasional & 1.171 & 75,1 & 74 & 6,3 & $\leq 0,001$ \\
\hline Abuso/Dependência & 389 & 24,9 & 60 & 15,4 & \\
\hline \multicolumn{6}{|l|}{ Álcool } \\
\hline Sem uso/Ocasional & 1.141 & 73,1 & 78 & 6,8 & $\leq 0,001$ \\
\hline Abuso/Dependência & 419 & 26,9 & 56 & 13,4 & \\
\hline \multicolumn{6}{|l|}{ Maconha } \\
\hline Sem uso/Ocasional & 1.487 & 95,3 & 115 & 7,7 & $\leq 0,001$ \\
\hline Abuso/Dependência & 73 & 4,7 & 19 & 26 & \\
\hline \multicolumn{6}{|l|}{ Cocaína } \\
\hline Sem uso/Ocasional & 1.527 & 97,9 & 124 & 8,1 & $\leq 0,001$ \\
\hline Abuso/Dependência & 33 & 2,1 & 10 & 30,3 & \\
\hline \multicolumn{6}{|l|}{ Crack } \\
\hline Sem uso/Ocasional & 1.544 & 98,1 & 128 & 8,3 & 0,001 \\
\hline Abuso/Dependência & 16 & 1,9 & 6 & 37,5 & \\
\hline \multicolumn{6}{|l|}{ Anfetamina } \\
\hline Sem uso/Ocasional & 1.545 & 99,0 & 128 & 8,3 & 0,001 \\
\hline Abuso/Dependência & 15 & 1,0 & 6 & 40,0 & \\
\hline \multicolumn{6}{|l|}{ Sedativo } \\
\hline Sem uso/Ocasional & 1.528 & 97,9 & 123 & 8,0 & $\leq 0,001$ \\
\hline Abuso/Dependência & 32 & 2,1 & 11 & 34,4 & \\
\hline Total & 1.560 & 100,0 & 134 & 8,6 & \\
\hline
\end{tabular}

Tabela 4

Comportamentos de risco à saúde, relacionados à sexualidade. Pelotas, Rio Grande do Sul, Brasil.

\begin{tabular}{|c|c|c|c|c|c|}
\hline & \multirow[t]{2}{*}{ n } & \multirow[t]{2}{*}{$\%$} & \multicolumn{2}{|c|}{ Risco de suicídio } & \multirow[t]{2}{*}{ Valor de $p$} \\
\hline & & & $\mathbf{n}$ & $\%$ & \\
\hline \multicolumn{6}{|c|}{ Uso de preservativo, na última relação sexual } \\
\hline Não & 594 & 42,0 & 62 & 10,5 & 0,025 \\
\hline Sim & 819 & 58,0 & 57 & 7,0 & \\
\hline \multicolumn{6}{|c|}{ Ingeriu bebida alcoólica, antes da última relação sexual } \\
\hline Não & 1.178 & 83,4 & 96 & 8,2 & 0,348 \\
\hline Sim & 234 & 16,6 & 24 & 10,3 & \\
\hline \multicolumn{6}{|l|}{ Parceiro fixo } \\
\hline Não teve relação & 138 & 8,9 & 38 & 10,2 & $\leq 0,001$ \\
\hline Não & 371 & 24,0 & 82 & 7,9 & \\
\hline Sim & 1.037 & 67,1 & 120 & 8,5 & \\
\hline \multicolumn{6}{|c|}{ Relação sexual com quantas pessoas, nos últimos 12 meses } \\
\hline Não teve relação & 902 & 57,9 & 61 & 6,8 & 0,018 \\
\hline 2-4 pessoas & 316 & 20,3 & 39 & 12,3 & \\
\hline 5 ou mais pessoas & 339 & 21,8 & 34 & 10,0 & \\
\hline Total & 1.560 & 100,0 & 134 & 8,6 & \\
\hline
\end{tabular}


que carregavam arma branca $(29,2 \%)$ e arma de fogo (55,6\%) entre os que apresentaram risco de suicídio. $\mathrm{O}$ acesso à arma branca e de fogo facilita no planejamento de suicídio, uma vez que o indivíduo com risco encontra-se vulnerável e, quando exposto a estímulos, possibilita o ato. Segundo o Ministério da Saúde, uma maneira de prevenção do suicídio é impedir o acesso aos meios de cometê-lo, como por exemplo, remover armas, faca, veneno 19 .

Estudos têm identificado o abuso de substâncias como fator associado ao comportamento suicida na juventude 20,21. Nos Estados Unidos, um estudo longitudinal com 1.200 jovens adultos examinou a associação entre o tabagismo e risco de suicídio, e como resultado, foi verificado que fumar diariamente foi relacionado ao risco de suicídio independentemente de transtornos mentais 20 . Outro estudo, realizado na Suíça, investigou a associação entre tabagismo e história de tentativas de suicídio em uma amostra de 180 adultos hospitalizados. O tabagismo foi significativamente associado com história de tentativas de suicídio 21 . Há uma escassez de dados na literatura que relacione risco de suicídio a outras drogas. Neste estudo estiveram associados ao risco de suicídio o abuso ou a dependência de tabaco $(15,4 \%)$, álcool (13,4\%), cocaína (30,3\%), crack $(37,5 \%)$, maconha $(26 \%)$, anfetamina (40\%) e sedativos (34,4\%). Estudos retrospectivos relatam não somente a presença de abuso ou dependência de álcool e outras drogas antes do incidente suicida, como também o aumento do consumo por ocasião do incidente ${ }^{22}$. Foi observado que entre os jovens com risco de suicídio, é alto o índice dos que faziam uso de crack e de anfetaminas. Tal dado é preocupante visto que o uso dessas substâncias vem aumentando nos últimos tempos, a primeira de forma ilícita e a segunda, lícita, na venda de medicamentos.

A relação encontrada entre uso de substâncias e risco de suicídio aponta uma das limitações deste estudo. Sabe-se que tanto o uso de substâncias quanto o risco de suicídio estão as- sociados a transtornos psiquiátricos. No mesmo sentido, o resultado encontrado sobre comportamento sexual de risco e risco de suicídio levanta a limitação, pois esse tipo de comportamento frequentemente está presente em psicopatologias como transtorno de humor bipolar e transtorno de personalidade borderline. Porém, como este estudo foi de caráter descritivo dos comportamentos de risco, optou-se por não controlar as variáveis sobre transtornos psiquiátricos, até mesmo pelo fato de serem colineares.

Avaliar risco de suicídio é bastante difícil, uma vez que é um comportamento humano bastante complexo, com múltiplas causas - incluindo biológicas - e componentes psicossociais 23,24. Outro aspecto que dificulta este tipo de estudo é a dificuldade em identificar se uma morte foi por suicídio ou acidental.

Mesmo com as limitações, cabe destacar que um dos pontos positivos deste estudo diz respeito à amostra com jovens de 18 a 24 anos, o que permite averiguar de uma forma precoce como os jovens lidam com os aspectos que podem colocar sua vida em risco, além de pesquisar sobre comportamentos inconscientes ainda tão pouco investigados. Entre outros pontos fortes, a avaliação do risco de suicídio permite que sejam desenvolvidas estratégias de prevenção para tais condutas de risco. Convém ressaltar também que a amostra populacional minimiza os vieses que poderiam estar presentes se fossem contemplados apenas aqueles casos que buscassem tratamento.

Por tratar-se de um estudo de delineamento transversal, não foi possível avaliar a relação causal, não sabendo se a conduta de risco precede o risco de suicídio ou vice-versa. Para avaliar tal relação, seria necessário realizar um estudo longitudinal. Todavia, este estudo alerta quanto à importância da observação de comportamentos de risco em jovens não apenas como uma característica própria da etapa, mas sim como um preditor para comportamentos mais graves como o risco de suicídio. 


\section{Resumo}

O objetivo foi avaliar risco de suicídio e comportamentos de risco em jovens. Estudo transversal na zona urbana de Pelotas, Rio Grande do Sul, Brasil, realizado por amostragem sistemática. Foram incluídos jovens de 18 a 24 anos, com capacidade cognitiva e que assinaram termo de consentimento. Foram aferidos risco de suicídio (MINI); comportamentos de risco (YRBSS); abuso/dependência de substâncias (ASSIST); e nivel socioeconômico (ABEP). Os dados foram analisados no programa SPSS. A amostra constituiu-se de 1560 jovens e a prevalência de risco de suicídio foi de $8,6 \%$. O risco de suicídio foi associado com: ter sofrido acidente com necessidade de ir ao pronto-socorro $(p=0,011)$, ter entrado em briga com agressão física $(p=0,016)$, ter carregado arma branca $(p=0,001)$ e arma de fogo ( $p \leq 0,001$ ), ter abuso/dependência de substâncias ( $p \leq$ $0,001)$, não ter utilizado preservativo na última relação sexual ( $p=0,025)$, não ter parceiro fixo $(p \leq 0,001)$ e ter relação sexual com cinco ou mais pessoas ( $p=$ 0,018). Jovens com comportamentos de risco também demonstram indícios de risco de suicídio.

Suicídio; Assunção de Riscos; Adulto Jovem

\section{Referências}

1. Werlang SG, Borges VR, Fensterseifer L. Fatores de risco ou proteção para a presença de ideação suicida na adolescência. Interam J Psychol 2005; 39:259-66.

2. Casey PR, Dunn G, Kelly BD, Birkbeck G, Dalgard OS, Lehtinen V, et al. Factors associated with suicidal ideation in the general population: five-centre analysis from the ODIN study. Br J Psychiatry 2006; 189:410-5.

3. Kaplan H, Sadock BF. Medicina psiquiátrica de emergência. In: Kaplan H, Sadock BF, organizadores. Compêndio de psiquiatria: ciências do comportamento e psiquiatria clínica. Porto Alegre: Editora Artmed; 1997. p. 960-81.

4. Feijó RB, Oliveira EA. Comportamento de risco na adolescência. J Pediatr (Rio J.) 2001; 77 Suppl 2:S125-34.

5. Arnett J. Reckless behaviour in adolescence: a developmental perspective. Dev Rev 1992; 12:339-73.

6. Bahls S-C. Aspectos clínicos da depressão em crianças e adolescentes. J Pediatr (Rio J.) 2002; 78:359-66.

7. Werlang BG, Botega NJ. Comportamento suicida. Porto Alegre: Editora Artmed; 2004.

\section{Colaboradores}

L. A. Quevedo, A. B. Carvalho e T. A. Cardoso participaram da revisão bibliográfica, análise estatística, elaboração do artigo e aprovação final para publicação. R. A. Silva colaborou na elaboração do projeto, coordenação geral do estudo, revisão bibliográfica, análise estatística e aprovação final para publicação. K. Jansen e L. C. Ores contribuíram na elaboração do projeto, coordenação do trabalho de campo, análise estatística, revisão bibliográfica e aprovação final para publicação. L. D. M. Souza participou na elaboração do projeto, coordenação do trabalho de campo, revisão bibliográfica e aprovação final para publicação. R. T. Pinheiro colaborou na revisão bibliográfica, elaboração do projeto, análises estatísticas e aprovação final para publicação.

\section{Agradecimentos}

Ao Conselho Nacional de Desenvolvimento Científico e Tecnológico (CNPq) pelo financiamento.
8. Jiang Y, Perry DK, Hesser JE. Adolescent suicide and health risk behaviors: Rhode Island's 2007 Youth Risk Behavior Survey. Am J Prev Med 2010; 38:551-5.

9. Ores LC. Prevalência de ideação suicida e fatores associados: estudo de base populacional com jovens entre 18 a 24 anos, Pelotas-RS [Dissertação de Mestrado]. Pelotas: Programa de Pós-graduação em Saúde e Comportamento, Universidade Católica de Pelotas; 2007.

10. Hawton K, van Heeringen K. Suicide. Lancet 2009; 373:1372-81.

11. Eaton DK, Kann L, Kinchen S, Shanklin S, Ross J, Hawkins J, et al. Youth risk behavior surveillance United States, 2009. MMWR Surveill Summ 2009; 59:1-142.

12. Henrique IFS, Micheli D, Lacerda RB, Lacerda LA, Formigoni MLS. Validação da versão brasileira do teste de triagem do envolvimento com álcool, cigarro e outras substâncias (ASSIST). Rev Assoc Med Bras 2004; 50:199-206. 
13. Amorim P. Mini International Neuropsychiatric Interview (MINI): validação de entrevista breve para diagnóstico de transtornos mentais. Rev Bras Psiquiatr 2000; 22:106-15.

14. Sheehan DV, Lecrubier Y, Sheehan KH, Amorim P, Janavs J, Weiller E, et al. The Mini-International Neuropsychiatric Interview (M.I.N.I.): the development and validation of a structured diagnostic psychiatric interview for DSM-IV and CID-10. J Clin Psychiatry 1998; 59 Suppl 20:22-33.

15. Associação Brasileira de Empresas de Pesquisa. Critério de classificação econômica Brasil. http:// www.abep.org/novo/Utils/FileGenerate.ashx?id= 197 (acessado em Mai/2008).

16. Hernetkski K, Keskinen E. Self-destruction in Finnish motor traffic accidents in 1974-1992. Accid Anal Prev 1998; 30:697-704.

17. McGirr A, Renaud J, Bureau A, Seguin M, Lesage A, Turecki G. Impulsive-aggressive behaviours and completed suicide across the life cycle: a predisposition for younger age of suicide. Psychol Med 2008; 38:407-17.

18. Departamento de Análise de Situação em Saúde, Secretaria de Vigilância em Saúde, Ministério da Saúde. Mortalidade por suicídio. In: Departamento de Análise de Situação em Saúde, Secretaria de Vigilância em Saúde, Ministério da Saúde, organizador. Saúde Brasil 2006: uma análise da situação de saúde no Brasil. Brasília: Ministério da Saúde; 2006. p. 565-86. (Série G. Estatística e Informação em Saúde).
19. Ministério da Saúde. Prevenção do suicídio: manual dirigido profissionais da saúde da atenção básica. Brasília: Ministério da Saúde; 2009.

20. Breslau N, Schultz LR, Johnson EO, Peterson EL, Davis GC. Smoking and the risk of suicidal behavior: a prospective study of a community sample. Arch Gen Psychiatry 2005; 62:328-34.

21. Keizer I, Gex-Fabry M, Eytan A, Bertschy G. Smoking in psychiatric inpatients: Association with working status, diagnosis, comorbid substance abuse and history of suicide attempts. Addict Behav 2009; 34:815-20.

22. Cavaiola AA, Lavender N. Suicidal behavior in chemically dependent adolescents. Adolescence 1999; 34:735-44.

23. Rihmer Z. Can better recognition and treatment of depression reduce suicide rates? A brief review. Eur Psychiatry 2001; 16:406-9.

24. Fu Q, Heath AC, Bucholz KK, Nelson EC, Glowinski AL, Goldberg J, et al. A twin study of genetic and environmental influences on suicidality in men. Psychol Med 2002; 32:11-24.

Recebido em 20/Abr/2011

Versão final reapresentada em 03/Ago/2011

Aprovado em 03/Out/2011 